\title{
Perfil lipídico: intervalos de referência em escolares de 2 a 9 anos de idade da cidade de Maracaí (SP)
}

\author{
Lipid profile: normal range values in school children from two to nine years old from the city of Maracaí, SP, Brazil
}

\author{
Mario Seki \\ Fabiana Petruscke Niyama² \\ Marisa Okabe Seki ${ }^{2}$ \\ Plínio Gomes Pereira Júnior ${ }^{3}$ \\ Matiko Okabe Seki ${ }^{4}$ \\ Ana Maria Bonametti ${ }^{5}$ \\ Tiemi Matsuo ${ }^{5}$ \\ Alexandre José Faria Carrilho ${ }^{5}$
}

\section{unitermos resumo}

Perfil lipídico

Intervalos de referência

Crianças

Colesterol

Triglicerídeos

Dislipidemias
Introdução: As recentes publicações disponíveis em nosso meio sobre dislipidemias na infância indicam progressão de uma silenciosa epidemia, que pode agravar as taxas de morbidade e mortalidade por doenças cardiovasculares nos próximos anos. Na falta de padronização dos intervalos de referência para população brasileira, muitos autores utilizam valores procedentes de outros países, em particular os recomendados pelas III Diretrizes Brasileiras Sobre Dislipidemias (III DBSD, 2001), que foram transcritos do National Cholesterol Education Program-NCEP (EUA, 1992). Objetivo: Estabelecer intervalos de referência para triglicerídeos (TG), colesterol total (CT) e frações em escolares de 2 a 9 anos de idade da cidade de Maracaí, SP. Métodos: delineamento transversal. Foram avaliados perfis lipídicos de 1.202 alunos de seis escolas (cinco públicas e uma privada), de 2 a 9 anos de idade, no período de 25 de março a 8 de outubro de 2002. Empregaram-se reações enzimáticas para determinações de TG e CT, kits Johnson \& Johnson, metodologia de química seca e auto-analisador Vitros 750. Utilizaram-se reagentes magnéticos (sulfato de dextran e cloreto de magnésio, Johnson \& Johnson) para precipitação de LDL-c e VLDL-c e subseqüente determinação enzimática de HDL-c. O LDL-c fol obtido através de cálculo de Friedewald para TG abaixo de $400 \mathrm{mg} / \mathrm{dl}$; valores superiores a este foram determinados com kits Merck CHOD-PAP e leitura fotométrica. Resultados: As exclusões totalizaram 266 casos e os analisados, 936. Os valores obtidos na amostra estudada diferem dos intervalos de referência recomendados pelas III DBSD. As maiores variações dos resultados de Maracaí, em relação às III DBSD, foram do percentil 95 de TG: $130 \mathrm{mg} / \mathrm{dl}$ (+ 30\%); percentil 75 de LDL-c: 101,1 mg/dl (-8,1\%); percentil 5 de HDL-c: $34 \mathrm{mg} / \mathrm{dl}$ (-15\%). Discussão: os intervalos de referência transcritos de outros países desconsideram diferenças étnicas, socioeconômicas e hábitos alimentares da população brasileira. Desta forma, estudos epidemiológicos que os utilizam podem conter indesejáveis vieses de mensuração. Na prática clínica, a utilização dos intervalos de referência de outras procedências, nessa faixa etária para TG, pode provocar investigações adicionais desnecessárias e danos irreparáveis ao desenvolvimento das crianças através de intervenções desastradas sob forma de dietas hipocalóricas ou, pior ainda, prescrição de medicamentos. Conclusão: Os intervalos de referência da amostra de escolares de Maracaí de 2 a 4 anos diferem dos recomendados pelas III DBSD. Este fato ressalta a necessidade de realização de estudos semelhantes em outras regiões para padronização brasileira dos intervalos de referência para TG, CT e frações. 


\section{Introdução}

A associação entre as dislipidemias - alterações metabólicas lipídicas, com repercussões sobre os níveis das lipoproteínas em circulação e nas concentrações dos seus diferentes componentes - e a aterosclerose está claramente estabelecida na literatura $(9,16,22)$.

A aterosclerose é uma afecção das artérias de grande e médio calibres caracterizada por lesões com aspectos de placas (ateromas); tem início insidioso a partir da infância, evolução lenta e silenciosa, e suas manifestações clínicas na vida adulta repercutem sob diversas condições mórbidas do aparelho circulatório que culminam nas elevadas taxas de mortalidade $(6,22)$.

As publicações atualizadas em nosso meio indicam avanço das dislipidemias em crianças e jovens $(5,8,18)$. Este fenômeno pode agravar os indicadores de saúde no futuro. Dados do Ministério da Saúde/Funasa registram que, no ano de $1998,32,6 \%$ da mortalidade por causas conhecidas foram devidos ao conjunto de doenças da CID10, que constituem as doenças do aparelho circulatório; entre estas, as doenças isquêmicas do coração responderam por $29,6 \%$ e as cerebrovasculares, por $33 \%$ (7).

Nos estudos nacionais, observa-se, além da falta de padronização, a utilização de intervalos de referência procedentes de outros países. De fato, inexistem valores adequados à nossa realidade, pois os recomendados pelas III Diretrizes Brasileiras Sobre Dislipidemias (III DBSD) foram transcritos do National Cholesterol Education Program (NCEP) $(9,16,22)$. Um potencial viés de mensuração em estudos epidemiológicos que utilizam esses valores, portanto, precisa ser ponderado, pois desconsideram múltiplas variáveis que incluem as diferenças étnicas, socioeconômicas e hábitos alimentares da população brasileira. Diante destas perspectivas, tornam-se necessários maiores estudos para retificar ou ratificar os valores recomendados para a realidade nacional.

O objetivo deste estudo é estabelecer intervalos de referência para triglicerídeos (TG), colesterol total (CT), colesterol-LDL (LDL-C), colesterol-HDL (HDL-C), e não-HDLcolesterol (não-HDL-c) em escolares de 2 a 9 anos de idade da cidade de Maracaí, SP.

\section{Casústica e métodos}

\section{População e amostra}

O censo demográfico 2000 do Instituto Brasileiro de Geografia e Estatística (IBGE) registrou 13.004 habitantes na cidade de Maracaí, sudoeste de São Paulo. A maioria dos trabalhadores exerce atividades profissionais na zona rural e na indústria sucroalcooleira local.

Os dados fornecidos pela Secretaria Municipal de Educação daquela cidade indicam uma população escolar de 1.247 alunos na faixa etária de 2 a 9 anos, matriculados nas seis escolas, cinco públicas e uma privada, das zonas urbana e rural (17).

No período de 25 de março a 8 de outubro de 2002, foram coletadas amostras biológicas de 1.202 escolares de 2 a 9 anos de idade, 591 do sexo feminino e 611 do masculino, aparentemente hígidos. Esta amostra representa $96,4 \%$ da população escolar nesta faixa etária. Os alunos participaram como voluntários do estudo mediante assinatura do pai ou do responsável no termo de consentimento, que faculta desistência do estudo a qualquer momento.

\section{Delineamento}

Estudo transversal.

\section{Mensuração}

Os protocolos contendo dados pessoais e familiares foram preenchidos por professores(as), inspetores(as) ou colaboradores(as), e os dados físicos e antropométricos, obtidos por auxiliares de enfermagem devidamente treinados para estas finalidades. A balança antropométrica foi aferida previamente pelo Instituto de Pesos e Medidas (Ipem) e os procedimentos para obtenção das medidas seguiram as padronizações da World Health Organ Tech Rep Serv 1995 (23). Foram tomadas duas medidas de peso e duas de altura, extraindo-se média aritmética simples, com os voluntários descalços e utilizando vestimentas leves. O intervalo das medições de peso foi de $0,1 \mathrm{~kg}$ e da altura, 0,1 cm. O índice de massa corpórea (IMC) foi obtido pela relação peso $(\mathrm{kg}) /$ altura $^{2}\left(\mathrm{~m}^{2}\right)$, e ajustado para sexo e idade pela curva dos percentis de acordo com Needlman e preconizado pelo National Center for Health Statistics (NCHS) (10). A classificação socioeconômica baseou-se nos critérios de Barros e Victora (3) e da Associação Nacional de Empresas de Pesquisa (2).

O controle de processo antes e após ensaios, que inclui coleta, centrifugação, armazenamento e remessa dos materiais biológicos, foi realizado de acordo com procedimentos documentados dos Laboratórios Dr. Joelson Ltda. S/C (Assis, SP) e Labmed/Labimagem (Londrina, PR), certificados pelo Sistema da Qualidade ISO 9002, respectivamente pela ABS Quality Evaluations e pela Fundação Carlos 
Alberto Vanzolini. Os cuidados para a fase pré-analítica seguiram orientações das III DBSD: dieta habitual e peso estável pelo menos duas semanas antes da coleta; jejum de 12 a 14 horas; repouso prévio, sentado, pelo menos cinco minutos antes da coleta; não ter praticado exercícios vigorosos na véspera e não ter ingerido bebidas alcoólicas. A separação do soro foi realizada dentro de três horas após a coleta do material biológico.

Os procedimentos analíticos foram realizados no Labmed, por reações enzimáticas para $\mathrm{TG}$ e $\mathrm{CT}$, utilizando-se kits Johnson \& Johnson (Ortho Clinical Diagnostics), metodologia de química seca, e auto-analisador Vitros 750. Utilizaram-se reagentes magnéticos (sulfato de dextran e cloreto de magnésio, Johnson \& Johnson) para precipitação de LDLc e VLDL-c e subseqüente determinação enzimática de HDLc. O LDL-c para TG sérico abaixo de $400 \mathrm{mg} / \mathrm{dl}$ foi obtido pelo cálculo de Friedewald: LDL-C = CT - HDL-c - TG/5 (16, 22); para valores superiores a este, os soros foram precipitados com sulfato de polivinilo dissolvido em polietilenoglicol a 25\%, pH 6,7 e posterior determinação do LDL-c com kits Merck CHOD-PAP e leitura fotométrica no aparelho CELM modelo E-225. O não-HDL-c foi calculado através da fórmula CT - HDL-c (19). Os controles de qualidade internos (diários) e externos (mensais) foram realizados, respectivamente, com soros do fabricante e do Programa de Excelência para Laboratórios Médicos (Pelm), da Sociedade Brasileira de Patologia Clínica.
As técnicas estatísticas utilizaram o programa Excel 2000, Windows 98, para obter valores dos percentis 5, 25, 50, 75, 90 e 95 para TG, CT, LDL-C, HDL-c e nãoHDL-C, estratificados conforme o sexo.

\section{Resultados}

Do total de 1.202 voluntários foram excluídos 266 $(22,1 \%)$ pelas seguintes razões: 205 por apresentarem IMC igual ou maior que o percentil 90, considerados com excesso de peso e obesos (21); 49 voluntários com IMC igual ou abaixo do percentil 5 , considerados atrasos de crescimento (10); três por irregularidades na coleta de dados ou materiais biológicos; três por uso de anticonvulsivantes, que podem interferir nos níveis lipídicos (22); três por antecedentes pessoais de hepatopatias, dois de tireopatia e um de diabetes, que podem apresentar dislipidemias secundárias a estas doenças (22). O grupo estudado de 936 escolares foi constituído por 475 do sexo masculino e 461 do feminino, média de 6,8 e mediana de 7 anos. A classificação socioeconômica revelou que 88 alunos $(9,4 \%)$ pertencem às classes sociais A e B; 404 (43,2\%), à classe C; 422 $(45,1 \%)$, à classe $D ;$ e $22(2,3 \%)$, à classe $E$.

Os valores obtidos conforme o sexo e os percentis 5 (P5), 25 (P25), 50 (P50), 75 (P75), 90 (P90) e 95 (P95) estão nas Tabelas 1 a 5.

\section{Valores de triglicerídeos ( $\mathrm{mg} / \mathrm{dl}$ ) conforme sexo e percentis na amostra de escolares}

Tabela 1 de 2 a 9 anos de idade da cidade de Maracaí (SP)

\begin{tabular}{|c|c|c|c|c|c|c|}
\hline \multirow[b]{2}{*}{ Sexo } & \multicolumn{5}{|c|}{ Triglicerídeos (mg/dl) } & \multirow[b]{2}{*}{ P95 } \\
\hline & P5 & P25 & P50 & P75 & P90 & \\
\hline Masculino $(n=475)$ & 40,7 & 57 & 69 & 87 & 103 & 125 \\
\hline Feminino ( $n=461$ ) & 45 & 61 & 75 & 93 & 117 & 135 \\
\hline Ambos os sexos $(n=936)$ & 43 & 58 & 72 & 90 & 112,5 & 130 \\
\hline
\end{tabular}

\section{Valores de colesterol total ( $\mathrm{mg} / \mathrm{dll}$ ) conforme sexo e percentis na amostra de escolares} Tabela 2 de 2 a 9 anos de idade da cidade de Maracaí (SP)

\begin{tabular}{lcccccc}
\hline & \multicolumn{7}{c}{ Colesterol total $(\mathrm{mg} / \mathrm{dl})$} \\
Sexo & P5 & P25 & P50 & P75 & P90 & P95 \\
\hline Masculino $(n=475)$ & 114 & 134,5 & 149 & 168 & 186 & 195 \\
Feminino $(n=461)$ & 117 & 138 & 154 & 173 & 185 & 196 \\
Ambos os sexos $(\mathbf{n = 9 3 6 )}$ & $\mathbf{1 1 4}$ & $\mathbf{1 3 6}$ & $\mathbf{1 5 2}$ & $\mathbf{1 7 0}$ & $\mathbf{1 8 6}$ & $\mathbf{1 9 6}$ \\
\hline
\end{tabular}


Valores de colesterol LDL ( $\mathrm{mg} / \mathrm{dl})$ conforme sexo e percentis na amostra de escolares

Tabela 3 de 2 a 9 anos de idade da cidade de Maracaí (SP)

\begin{tabular}{lcccccc}
\hline & \multicolumn{7}{c}{ Colesterol LDL $(\mathrm{mg} / \mathrm{dl})$} & & P95 \\
Sexo & P5 & P25 & P50 & P75 & P90 & P95 \\
Masculino $(\mathrm{n}=475)$ & 49,2 & 71,5 & 82,8 & 100,8 & 118,2 & 126,1 \\
Feminino $(\mathrm{n}=461)$ & 49,4 & $\mathbf{7 0 , 8}$ & 87,2 & 101,4 & 115,6 & 122,8 \\
Ambos os sexos $(\mathbf{n = 9 3 6 )}$ & $\mathbf{4 9 , 2}$ & $\mathbf{7 1 , 2}$ & $\mathbf{8 4 , 6}$ & $\mathbf{1 0 1 , 1}$ & $\mathbf{1 1 7 , 6}$ & $\mathbf{1 2 4 , 7}$ \\
\hline
\end{tabular}

\section{Valores de colesterol HDL ( $\mathrm{mg} / \mathrm{dl})$ conforme sexo e percentis na amostra de escolares} Tabela 4 de 2 a 9 anos de idade da cidade de Maracaí (SP)

\begin{tabular}{|c|c|c|c|c|c|c|}
\hline \multirow[b]{2}{*}{ Sexo } & \multicolumn{5}{|c|}{ Colesterol HDL (mg/dl) } & \multirow[b]{2}{*}{ P95 } \\
\hline & P5 & P25 & P50 & P75 & P90 & \\
\hline Masculino ( $n=475$ ) & 33 & 43 & 50 & 57 & 65 & 68,3 \\
\hline Feminino $(n=461)$ & 34 & 44 & 50 & 57 & 66 & 73 \\
\hline Ambos os sexos $(n=936)$ & 34 & 44 & 50 & 57 & 65 & 70,3 \\
\hline
\end{tabular}

\section{Valores de não-HDL-c (mg/ dl) conforme sexo e percentis na amostra de escolares}

Tabela 5 de 2 a 9 anos de idade da cidade de Maracaí (SP)

\begin{tabular}{|c|c|c|c|c|c|c|}
\hline \multirow[b]{2}{*}{ Sexo } & \multicolumn{5}{|c|}{ Não-HDL-c (mg/ dl) } & \multirow[b]{2}{*}{ P95 } \\
\hline & P5 & P25 & P50 & P75 & P90 & \\
\hline Masculino $(n=475)$ & 61,7 & 85 & 99 & 117 & 135 & 145 \\
\hline Feminino $(n=461)$ & 64 & 87 & 103 & 119 & 134 & 143 \\
\hline Ambos os sexos $(n=936)$ & 63 & 86 & 101 & 118 & 134,5 & 144 \\
\hline
\end{tabular}

Os resultados obtidos com valores iguais ou maiores que os percentis 75 e menores que os 95 foram considerados limítrofes para CT e LDL-c; valores iguais ou maiores que os percentis 95 , considerados elevados para TG, CT e LDL-C; e valores iguais ou menores que os percentis 5, baixos para HDL-C (14).

Os intervalos de referência estabelecidos para a amostra de escolares de ambos sexos da cidade de Maracaí até 9 anos de idade, conforme os percentis determinados por Rifking (14), os valores recomendados pelas III DBSD (22) e a comparação dos resultados obtidos com as III DBSD, em porcentagem de variação, estão na Tabela 6.

\section{Discussão}

A utilização dos intervalos de referência para lípides e lipoproteínas, com finalidade de distinguir populações normais das anormais, principalmente para aqueles abaixo do valor da média e livres de riscos para doenças cardiovasculares, pode ser questionada devido à distribuição contínua dos seus níveis plasmáticos (14). Os intervalos de referência, entretanto, continuam sendo utilizados para identificar pessoas com alto risco para desenvolver doenças cardiovasculares em comparação com pessoas do mesmo sexo e idade com baixos níveis dos lípides (14).

Uma das dificuldades para estabelecer intervalos de referência é a definição de população clinicamente normal. Rocha e Andriolo consideram, nesta categoria, pessoas de ambos sexos em condições habituais de atividade física e dieta, supostamente livres de qualquer anormalidade óbvia (15). Apesar de não haver padronização dos critérios de definição desta categoria, ponderando a natureza deste estudo, foram excluídos 49 casos com IMC igual ou menor ao percentil 5, considerados 
Intervalos de referência estabelecidos para amostra de escolares de 2 a 9 anos de idade da cidade de Maracaí (ambos os sexos) conforme os percentis estabelecidos, os valores Tabela 6 recomendados pelas III DBSD e porcentagem de variação

\begin{tabular}{lcccc}
\hline Analito & Percentil & $\begin{array}{c}\text { Intervalos de referência } \\
\text { de Maracaí }(\mathrm{mg} / \mathrm{dl})\end{array}$ & $\begin{array}{c}\text { Valores das III } \\
\text { DBSD (mg/ dI) }\end{array}$ & \% variação \\
\hline TG & 95 & 130 & 100 & +30 \\
CT & 75 & 170 & 170 & 0 \\
& 95 & 196 & 200 & -2 \\
LDL-C & 75 & 101,1 & 110 & $-8,1$ \\
& 95 & 124,7 & 130 & $-4,1$ \\
HDL-c & 5 & 34 & 40 & -15 \\
Não-HDL-c & 75 & 118 & $*$ & $* *$ \\
& 95 & 144 & $*$ & $* *$ \\
\hline
\end{tabular}

*Valores não-estabelecidos; **não-avaliada.

atraso de crescimento (10). As exclusões dos voluntários com IMC igual ou maior ao percentil 90, definidos como excesso de peso e obesos (21), que totalizaram 205 casos, foram devidas às associações destes fatores com as dislipidemias $(9,21,22)$.

Considerando que os intervalos de referência recomendados pelas III DBSD (22) são adotados por diversos autores $(1,6,12,13)$, este estudo procurou utilizá-los para análise comparativa com os resultados obtidos. Assim, a delimitação da idade de 2 a 9 anos teve a finalidade de permitir comparação entre os valores obtidos e os intervalos de referência recomendados pelas III DBSD para esta mesma faixa etária $(16,22)$. Não há indicações para estudo do perfil lipídico em crianças menores de 2 anos (13). Estudo comparativo em relação a sexo não foi realizado devido à ausência desta estratificação nas III DBSD (22). As características multirraciais e a miscigenação da população brasileira dificultaram a coleta de dados e prejudicaram a análise dos voluntários e a sua estratificação em relação à etnia ou à cor da pele.

A classificação socioeconômica do grupo estudado revelou uma pirâmide social encontrada comumente na população brasileira. A maioria da casuística pertence às classes $C$, D e E $(90,6 \%)$, e pequena parcela, às classes A e $B(9,4 \%)$. Esta classificação socioeconômica, heterogênea fornece importante subsídio sobre a amostra estudada, permitindo utilização de seus dados em estudos populacionais com condições similares.

O estudo da associação entre os valores encontrados e os hábitos alimentares, apesar de desejável, não pode ser analisado. Dacas et al., entretanto, não encontraram relação entre hábitos alimentares, obtida através de inquéri- tos, com níveis séricos de lípides e lipoproteínas em crianças e jovens (4).

Neste estudo, foram adotados percentis estabelecidos nos estudos de Rifkind e Segal, do Lipid Research Clinics Program (14). Os valores dos percentis 5 para CT e LDL-C podem ser úteis para detectar raros casos com deficiências de lipoproteínas (14). O uso do percentil 75 amplia a proporção de indivíduos que merecem cuidados por correrem maiores riscos de ser acometidos por doenças cardiovasculares atribuídas a hipercolesterolemia (14). Para estabelecer intervalos de referência para TG, considerou-se apenas o percentil 95, porque a relação independente deste analito com doenças cardiovasculares não está bem estabelecida $(14,20)$.

Apesar de não existirem evidências da relação de TC com doenças ateroscleróticas, seus níveis séricos são considerados importantes para a avaliação das dislipidemias. Além disso, deve ser ponderada a sua participação no cálculo de Friedewald para LDL-c $(16,22)$. Os valores de VLDL-c não são apresentados neste estudo, pois, formalmente, os distúrbios de hipertrigliceridemias são realizados através dos níveis séricos de TG (14).

Dos analitos do perfil lipídico, as maiores atenções estão voltadas para as concentrações séricas de LDL-C, em função da sua participação na gênese das células espumosas que constituem as placas gordurosas (22). Estudos recentes, entretanto, consideram não-HDL-c (CT - HDL-c) o melhor indicador de doenças cardiovasculares por incluir todas as classes de lipoproteínas aterogênicas (19). Os valores encontrados de não-HDL-c revelaram-se maiores que os de LDL-c, e a sua comparação em relação às III DBSD ficou prejudicada por falta de sua aplicação na diretriz brasileira (22). 
As investigações epidemiológicas do perfil lipídico em crianças assumem importância cada vez maior diante das evidências do início do processo aterosclerótico na infância e das tendências de as dislipidemias persistirem na vida adulta $(9,16,22)$. Os estudos nacionais contribuem para avaliar o estágio das dislipidemias infanto-juvenis em nosso meio $(5,8,18)$. Entretanto a falta de padronização e a utilização dos intervalos de referência transcritos de outros países podem conter indesejáveis vieses de mensuração.

Os resultados obtidos do $\mathrm{CT}$ revelam compatibilidade do P75 em relação às III DBSD, e variação de $-2 \%$ do P95 em relação às III DBSD (Tabela 6). Maiores variações dos resultados obtidos da população escolar de Maracaí até 9 anos de idade, em relação às III DBSD, foram observadas no P95 de TG (variação de + 30\%), P75 de LDL-c (-8,1\%), P95 de LDL-c $(-4,1 \%)$ e P5 de HDL-C (-15\%). Estas diferenças podem ser devidas às influências de múltiplas variáveis, como constituições étnicas, níveis socioeconômicos, condições nutricionais, hábitos alimentares e estilos comportamentais de vida - provavelmente de baixa atividade física. Valores elevados para TG também foram observados por Moura et al. em Campinas, São Paulo (8). Os resultados apresentados sugerem que estudos epidemiológicos nacionais que utilizam intervalos de referência estrangeira podem conter vieses de mensuração na utilização dos valores dos P95 de TG, P75 e P95 de LDL-c e P5 de HDL-c.

Maiores atenções devem ser dispensadas aos resultados do percentil 95 de TG, de $130 \mathrm{mg} / \mathrm{dl}$, variação de + $30 \%$ em relação às III DBSD, fixada no limite de $100 \mathrm{mg} /$ dl para essa faixa etária (Tabela 6). Interpretações inadvertidas sobre intervalos de referência podem conduzir a investigações desnecessárias de hipertrigliceridemias factícias. Não podem ser desconsideradas as possibilidades de intervenções clínicas desastrosas sob forma de dietas hipocalóricas ou prescrição de medicamentos, com eventuais prejuízos irreparáveis no desenvolvimento de crianças dessa faixa etária. Olson considera exageradas as intervenções alimentares em crianças dislipidêmicas, pois, além de hiporresponsivas às dietas, as lesões gordurosas são inócuas na infância (11).

Estudos subseqüentes serão necessários para avaliar intervalos de referência para outras faixas etárias de interesse.

\section{Conclusão}

Os valores estabelecidos como intervalos de referência dos escolares de ambos sexos, de 2 a 9 anos de idade para TG, CT (P95), LDL-c e HDL-C diferem dos valores recomendados pelas III DBSD. Considerando-se os resultados obtidos, há necessidade de estudos similares em outras regiões para estabelecer intervalos de referência nacionais para os analitos do perfil lipídico.

\section{Agradecimentos}

Agradecemos as inestimáveis colaborações de Ortho Clinical Diagnostics (Johnson \& Johnson), Noely Yukiko Igawa Ribeiro, Antonio da Silva Cavalheiro, Doralice Franco Correia, Claury Alves da Silva, Benedito Salatini, Aguinaldo Oliveira Cruz, Eduardo Garcia da Silveira Filho, Sandra Albertini, Otávio Haguida Jr., Labmed/Labimagem Diagnóstico Avançado em Medicina (Londrina, PR), Laboratório Dr. Joelson (Assis, SP) e Labmar (Maracaí, SP), que possibilitaram a realização deste estudo.

\section{Referências}

1. Andriolo, A. Lipídeos. In: Carraza, F.R; A ndriolo, A. Diagnóstico laboratorial em pediatria. São Paulo: Sarvier, 2000. p. 99104.

2. A ssociação N acional de Empresas de Pesquisa. D isponível em <http://www.anep.gov.br> (acessado em 06/7/2002).

3. Barros, F.C . \& Victora, C.G. Epidemiologia da saúde infantil. São Paulo: Hucitec, 1998. p. 43-52.

4.D acas, P. et al. D eterminação dos padrõ es normais de colesterol total, triglicerídeos e frações - estudo piloto gama - prevenção de doenças cardiovasculares em adolescentes de C uritiba. Rev M ed São Paulo, 75(4): 198-214, 1996.

5. Gerber, Z.R.S. \& Zielinsky, P. Fatores de risco de aterosclerose na infância. Um estudo epidemiológico. Arq Bras Cardiol, 69(4):231-6, 1997.

6. Giannini, S.D. Aterosclerose. Dislipidemias. São Paulo: BG Cultural, 1998. 158p.

7. Ministério da Saúde. Fundação N acional de Saúde (Funasa). D isponível em <http:www.funasa.gov.br> (acessado em 20/8/2002)

8. Moura, E.C . et al. Perfil lipídico em escolares de Campinas, SP, Brasil. Rev Saúde Pública, 34(5): 499-505, 2000.

9. $\mathrm{N}$ ational Cholesterol Education Program (N CEP).The expert panel on blood cholesterol levels in children and adolescents. Pediatrics, 89 (suppl. 3): 525-70, 1992.

10. N eedlman, R.D. A valiação do crescimento. D erivação e interpretação da curva de crescimento. In: N elson, W .E. (ed.) Tratado de pediatria. 15. ed. Rio de Janeiro: Guanabara Koogan, 1997. p. 73-7. 
11. O Ison, R.E. Atherogenesis in children: implications for the prevention of atherosclerosis. Advances in Pediatrics, 47:55$78,2000$.

12. Rabelo, L.M. et al. D islipidemia em crianças e adolescentes. In: Martinez, T.L.R. Condutas clínicas nas dislipidemias. Belo Horizonte: Health, 1997. p. 205-13.

13. Rabelo, L.M .; Fisberg, M \& \& Martinez,T.L.R. D islipidemia na infância e na adolescência. In: Carraza, F.R. \& Andriolo, A. Diagnóstico laboratorial em pediatria.São Paulo:Sarvier,2000. p. 105-8.

14. Rifkind, B.M.\& Segal, P.Lipid research clinics program reference values for hyperlipidemia and hypolipidemia.JAM A,250(14): 1869-72, 1983

15. Rocha, M.H. \& A ndriolo, A. Introdução. In: Carraza, F.R. \& A ndriolo,A. Diagnóstico laboratorial em pediatria. São Paulo: Sarvier, 2000. p. 3-7.

16. Segundo consenso brasileiro so bre dislipidemias.D etecção, avaliação e tratamento. Arq Bras Cardiol,63(supl.): 1-16, 1.996.

17. Secretaria Municipal da Educação - Prefeitura Municipal de Maracaí. Número total de escolares que freqüentam as escolas em 15 de março de 2002. D ocumento interno; 12 de novembro de 2002.

18. Seki, M . et al. Estudo do perfil lipídico em crianças e jovens até 19 anos de idade.J Bras Patol, 37(4): 247-51, 2001.

19.Srinivasan,S.R.;M yers,L.\& Berenson, G.S.D istribution and correlates of non-high-density lipoprotein cholesterol in children: the Bogalusa Heart Study. Pediatrics, 110(3): e29, 2002.

20.Stein,E.A.\& Myers, G.L.N ational cholesterol education program recommendations for triglyceride measurement: executive summary. Clin Chem, 41(10): 1421-6, 1995.

21. Schonfeld-W arden, N . \& W arden, CH. Pediatric obesity. An overview of etiology and treatment. Pediatr Clin N orth Am, 44(2): 339-61, 1997.

22. III Diretrizes brasileiras sobre dislipidemias e diretriz de prevenção da aterosclerose do D epartamento de A terosclerose da Sociedade Brasileira de Cardiologia.Arq Bras Cardiol São Paulo, 77(supl. III): 4-48, 2001.

23. W orld Health 0 rganization. Physical status: the use and interpretation of anthro pometry. W orld Health Organization Technical Report Series Geneva, 854: 1-452, 1995. 